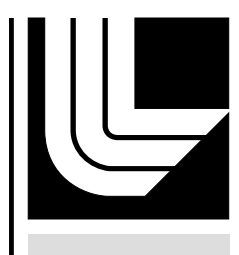

LAW RENCE LIVERMORE N A TIO N A L LABORATORY

\title{
UCRL-TR-215600
}

\section{Radiation Detection Center on the Front Lines}

A. Hazi

September 22, 2005 
This document was prepared as an account of work sponsored by an agency of the United States Government. Neither the United States Government nor the University of California nor any of their employees, makes any warranty, express or implied, or assumes any legal liability or responsibility for the accuracy, completeness, or usefulness of any information, apparatus, product, or process disclosed, or represents that its use would not infringe privately owned rights. Reference herein to any specific commercial product, process, or service by trade name, trademark, manufacturer, or otherwise, does not necessarily constitute or imply its endorsement, recommendation, or favoring by the United States Government or the University of California. The views and opinions of authors expressed herein do not necessarily state or reflect those of the United States Government or the University of California, and shall not be used for advertising or product endorsement purposes.

This work was performed under the auspices of the U.S. Department of Energy by University of California, Lawrence Livermore National Laboratory under Contract W-7405-Eng-48. 


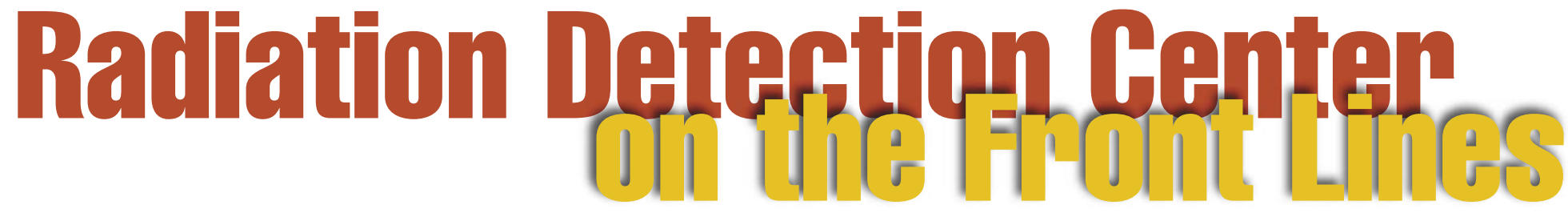

F you think a device that resembles a

cellular phone but detects a potential nuclear threat and transmits a description of the nuclear material to every nearby crisis center sounds like something out of a James Bond movie, you are in for a surprise. Since the 1930s, when scientists first used the Geiger counter, radiation detection equipment has gone through an amazing evolution in size, sensitivity, deployability, and power.

Lawrence Livermore, which has been developing radiation-related technologies for decades, continues to adapt radiation detection devices for national security needs. In 1999, the Laboratory began forming its Radiation Detection Center (RDC), a multidisciplinary organization that centralizes Livermore's radiation detection efforts. The RDC is supported through a memorandum of understanding between eight Laboratory directorates. Recently, several new radiation detection projects were funded by the Department of Homeland Security (DHS), which is expected to invest more than a billion dollars this year in science and technology across the nation to help the country detect and respond to terrorist threats.

The RDC helps to initiate and support many projects throughout the Laboratory that are developing new technology for national security and basic science programs within DHS, the Department of Energy (DOE), the Defense Threat Reduction Agency (DTRA), the Defense Advanced Research Projects Agency, the National Institutes of Health, the National

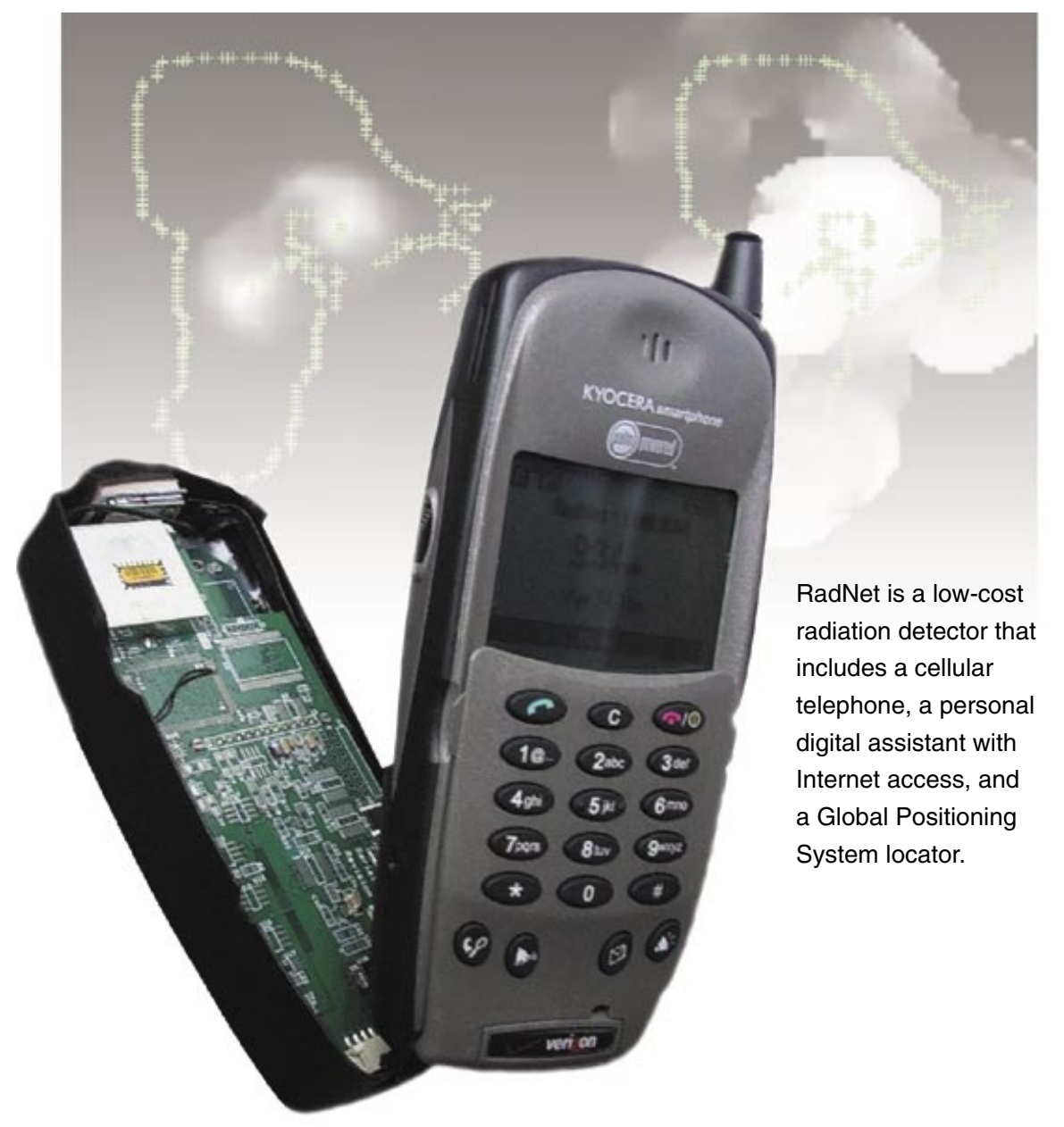

Aeronautics and Space Administration (NASA), and others. In addition, the center serves as an institutional resource for these organizations.

Many of today's radiation detection tools were developed in the 1960s. For years, the Laboratory's expertise in radiation detection resided mostly within its nuclear test program. When nuclear testing was halted in the 1990s, many of Livermore's radiation detection experts were dispersed to other parts of the Laboratory, including the directorates of Chemistry and Materials Science (CMS); Physics and Advanced Technologies (PAT); Defense and Nuclear Technologies (DNT); and Nonproliferation, Arms Control, and International Security (NAI).

The RDC was formed to maximize the benefit of radiation detection technologies being developed in 15 to 20 research and development $(\mathrm{R} \& \mathrm{D})$ programs. These efforts involve more than 200 Laboratory employees across eight directorates, in areas that range from electronics to computer simulations. The RDC's primary focus is the detection, identification, and analysis of nuclear materials and weapons. A newly formed outreach program within the RDC is responsible for conducting radiation detection workshops and seminars across the country and for coordinating university student internships.

Simon Labov, director of the RDC, says, "Virtually all of the Laboratory's programs use radiation detection devices in some way. For example, DNT uses radiation detection to create radiographs for their work in stockpile stewardship and in diagnosing explosives; CMS uses it to develop technology for advancing the detection, diagnosis, and treatment of cancer; and the Energy and Environment Directorate uses radiation detection in the Marshall Islands 
to monitor the aftermath of nuclear testing in the Pacific. In the future, the National Ignition Facility will use radiation detection to probe laser targets and study shock dynamics."

\section{Sorting the Signals}

One of the challenges facing nuclear researchers today is developing "smarter" detection systems that distinguish between radiation emissions from legitimate sources (for example, medical isotopes) and those from threatening sources. Nuclear materials, whether used in weapons or cancer therapy, typically emit gamma radiation. Many radioactive isotopes emit a unique spectrum of gamma rays that can penetrate substantial amounts of ordinary matter without being scattered or absorbed as visible light would be. These gamma rays can be analyzed to indicate the specific type of nuclear material.

\section{Radiation All around Us}

Radiant energy exists in two forms - ionizing and nonionizing. Ionizing radiation has sufficient energy to remove electrons from neighboring atoms, thereby creating charged particles (ions or radicals) in materials it strikes. An effect from such ionization can be biological damage at the molecular and cellular level. Nonionizing radiation, such as laser light and microwaves, does not have enough energy to remove electrons from neighboring atoms. The only observed biological effects of nonionizing radiation are heating effects.

The common types of ionizing radiation are alpha, beta, gamma, neutron, and $\mathrm{x}$ radiation. Some atoms, for example uranium and thorium, are naturally radioactive. Other radioactive isotopes, for example tritium and iodine-131, are made in reactors or accelerators.

Radiation deposited in a material, such as may occur in an experiment, is called a radiation dose. Dose equivalent is the term used when determining the effect on humans. Dose equivalent is measured in rems or sieverts. The total number is based on the radiation dose and type and whether the exposure was internal or external. Because the majority of radiation exposures is small, the unit of one-thousandth of a rem, or 1 millirem, is commonly used.

Background ionizing radiation (that is, radiation from natural sources) measures about 300 millirem per year in the U.S. The radiation comes from cosmic rays, radioactive material in the earth, naturally occurring radionuclides, such as potassium- 40 in food, and radon gas present in the air we breathe. Sources of human-caused ionizing radiation contribute an additional dose of approximately 70 millirem per year and include medical procedures, consumer products, and fallout radiation from the era of aboveground nuclear testing.

Exposure to large amounts of ionizing radiation (on the order of hundreds of times the natural exposure levels) increases the risk of cancer and genetic mutations that can be passed on to future generations. The extent of cell damage depends on the total amount of energy absorbed, the time period and dose rate of exposure, and the organs exposed.

In 1928, the International Commission on Radiological Protection-an independent, nongovernmental expert body - was established to recommend the maximum radiation dose to which people could be exposed. The commission set the level to not exceed 5 rem (5,000 millirem) per year. That limit is still used for radiation workers.

Most of the exposure associated with human activity is low-dose radiation. Although low-dose radiation does result in some damage to living tissue, the body can repair the damage. Recent research has indicated that low-dose ionizing radiation may activate protective and repair mechanisms and offer protection to the cells from a subsequent high dose of ionizing radiation. (See $S \& T R$, July/August 2003, pp. 12-19.) The model used for setting radiation protection limits is based on the risk being proportional to any radiation exposure above zero and is thus called the linear, no-threshold model.

The problem with early radiation detection systems is that they detected gamma rays from all radioactive material and could not distinguish between isotopes. Radioactive material is everywhere-from the concrete in our streets to the food we eat. Americium-241 is used in smoke detectors; cobalt-60 is used in medical treatment equipment; and potassium-40, a naturally occurring isotope in soil, is found in fruits and vegetables. (See the box below.)

Livermore scientists and engineers have made significant advancements in radiation detection equipment. Isotopes are now more easily distinguishable, reducing the confusion between threatening and nonthreatening sources. "We don't know exactly how a terrorist will build a device," says Labov. "But now we have more sophisticated instruments that have better spectral resolution. These instruments help us to identify common and legitimate radioactive materials, which increases our sensitivity to possible threats." Specialized integrated circuits and microelectronics, improved computer codes, and advancements in detector materials have made these instruments possible.

How many types of detection devices are needed? Labov says, "Several types are necessary because the needs vary depending on the field. A customs agent at a U.S. maritime port will benefit from cargo interrogation systems as well as radiation detectors placed in buoys, while a firefighter responding to a potential dirty bomb threat might benefit from a high-resolution, handheld detector. If smuggled nuclear material is intercepted, an ultrahighresolution gamma-ray spectrometer may help identify the origin of the material." (See S\&TR, January/February 2004, pp. 19-22; May 2004, pp. 12-15.)

Detection devices also vary in cost. The price tag for a large, laboratory-type spectrometer is $\$ 50,000$ to $\$ 80,000$, whereas a handheld detector may cost as little as $\$ 2,000$. In some cases, the low-cost detector may be more appropriate for a particular task. "For example," says physicist 
Bill Craig, who leads the Advanced Detector Group in PAT, "when you don't know the location of a threat, the dispersion of many cheaper detectors may be more efficient so that first responders can cover more area."

\section{Detector Makes Phone Calls}

The recently developed RadNet detector is both inexpensive (about \$2,000) and easily dispersed. This handheld instrument combines a cellular telephone, a personal digital assistant with Internet access, and a global positioning system locator with a radiation sensor. A number of RadNet units could be deployed as part of a wide-area network. Data collected by the units could be transmitted and plotted to a geographic map. In this way, law enforcement or other personnel could find the exact location of high-radiation signals from possible clandestine nuclear materials or devices.

In addition to being lightweight and able to operate at low power, each RadNet unit has sufficient energy resolution to eliminate alarms from background radiation emitted by such sources as food, medical devices, and soil. When it is not measuring specific radioactive samples, a RadNet unit monitors the ambient radiation field and communicates with a central processing (a)

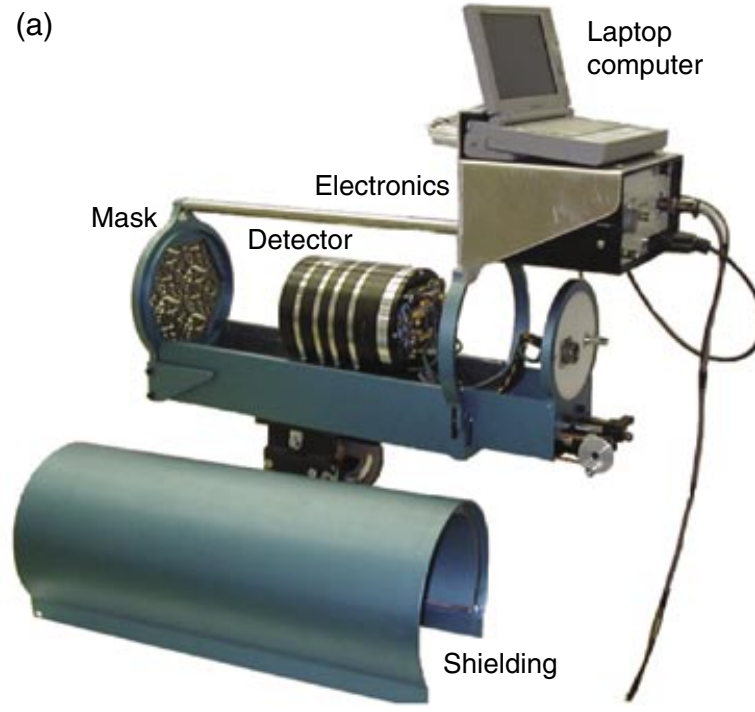

(c)

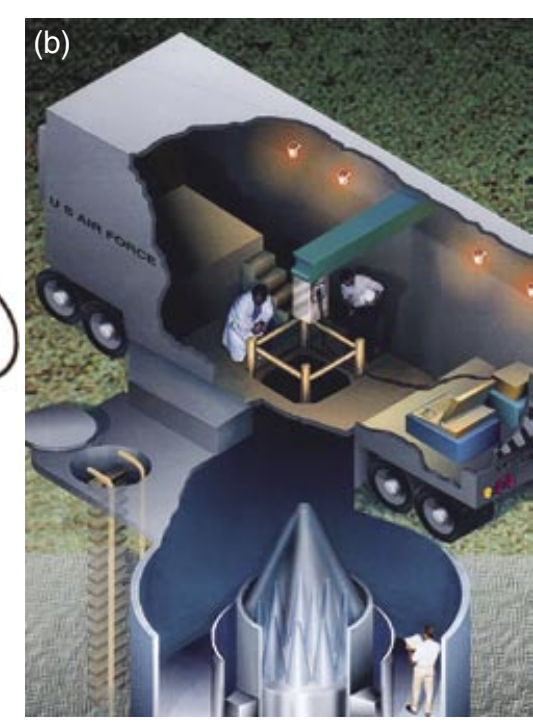

(a) The gamma-ray imaging spectrometer (GRIS) takes pictures of the high-energy gamma radiation given off by nuclear materials. The images are encoded on the detector by using a coded aperture mask that allows the user to recover the image through a computer program. (b) One potential application of GRIS is for nonintrusive treaty inspections. The drawing shows how the instrument might be used to inspect a multiple-warhead missile. (c) The image of a multiplewarhead missile taken with GRIS clearly shows each of the ten warheads. system in real time. "RadNet is a detection device that first responders, customs agents, and border inspectors can carry and use routinely because of its other features, such as a cellular phone," notes Labov.

The RDC is using a RadNet prototype in demonstrations to customs officials, fire departments, and other first responders. "Customs officials," says Craig, "have had radiation pagers for some time. However, those pagers cannot identify the radiation source or send information back to someone who can analyze the data."

The RadNet project combined resources from directorates across the Laboratory, which is what the RDC management likes to see. "RadNet's detector technology came from the astrophysics program in PAT," says Labov. "Engineering supplied electronics expertise for processing the detector's signals. CMS lent its expertise in detector material, and NAI and Engineering provided expertise in analyzing gamma-ray signatures to identify nuclear isotopes."

\section{Leave No Gamma Ray Behind}

Researchers have also made advances in semiconductor-based, gamma-ray imaging detectors. These imagers use increased sensitivity and spatial resolution to detect weak radioactive sources that would otherwise be masked by background gamma-ray emissions. Gamma-ray imagers are particularly useful when searching for lost, stolen, or hidden nuclear material in a large area. DOE, DHS, and DTRA are interested in Livermore's research on gamma-ray imaging because they are the agencies likely to be called if such a search is necessary.

Livermore is developing several gamma-ray imagers, each having different capabilities. One imager, called the gamma-ray imaging spectrometer (GRIS), can take gamma-ray "pictures" of the highenergy radiation emitted by nuclear materials. (See $S \& T R$, October 1995, pp. 14-26.) This instrument is useful for a variety of applications, including treaty 
inspections, mapping radioactive contamination, and determining what is inside a suspect object. Because gamma radiation is so difficult to focus, the instrument uses an imaging technique originally developed for high-energy astrophysics. The images are encoded on the detector by placing a sheet of material opaque to the radiation in front of the detector. The sheet is pierced with a carefully selected hole pattern that allows researchers to mathematically recover the image with a simple computer program. The system is about half the size of a personal computer.

GRIS was first developed for use in treaty inspections to monitor the location of nuclear missile warheads in a nonintrusive manner. In addition to its use in counterterrorism applications, GRIS is also expected to be useful in space to search for distant black holes and in hospitals to better detect, diagnose, and treat cancer.

Another version of GRIS being developed, the large-area imager, will be suited for longer-range searches. The largearea imager-approximately the size of a sofa - will be mounted in a small truck and capable of picking out weak radioactive sources from as far away as 100 meters.

The Compton camera is yet another type of gamma-ray imager under development. In addition to taking gamma-ray pictures, this imager should be able to identify very weak and typically invisible gamma-ray sources. The Compton camera operates without a mask or collimator, which can block many of the gamma rays emitted from a source. Instead, gamma rays coming from all directions at once are tracked as they scatter inside the detector. The camera's omnidirectional sensitivity is significantly higher than that of other imaging systems. Mathematical algorithms are used to retrace the paths of the gamma rays within the detector, and the results reveal the direction of the source.

Livermore's work on the Compton camera was originally funded through the
Laboratory Directed Research and Development Program and later by DOE. Today, DHS is funding a Livermore effort to develop a compact, potentially portable Compton camera. The main goal for the camera is to detect clandestine nuclear materials. However, the instrument could also be used to detect cancer early by using radiolabeled tracers to target unique molecular characteristics of the disease. A field-deployable prototype of the Compton camera is still a few years away. Laboratory researchers continue to test detector materials and determine the best size for the instrument.
Livermore physicist Kai Vetter says, "We are developing various types of gamma-ray imaging systems. Individuals who work in the field, searching for nuclear materials or carrying out stockpile stewardship activities, will tell us which system best suits their needs."

\section{Uncovering Hidden Elements}

Another radiation detection instrument is a spectrometer that can detect light elements, such as oxygen, within a heavy matrix, such as plutonium. While gammaray spectrometers are ideal for certain applications, in many situations, they
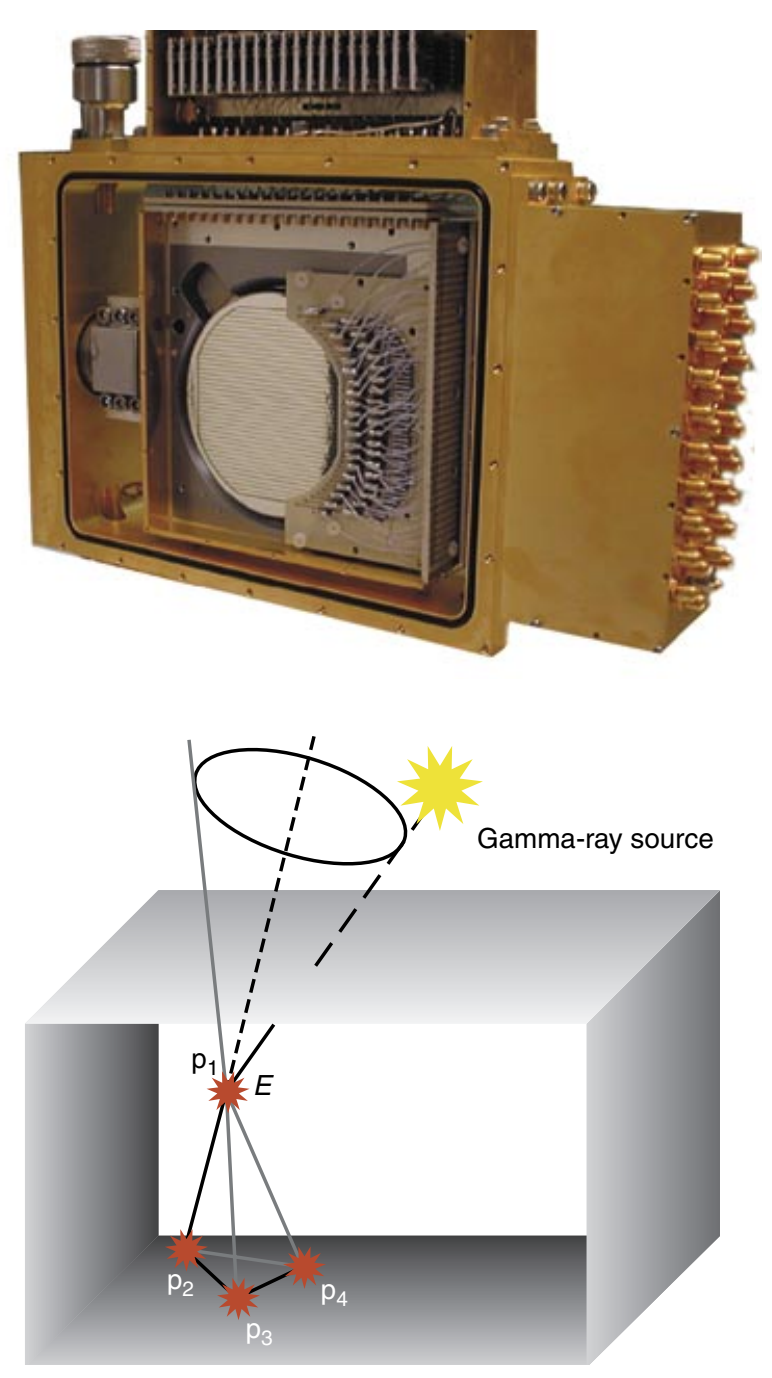

A two-dimensional, segmented germanium detector is one type of Compton camera. In the center is a high-purity germanium crystal. The detector is connected to individual cables and preamplifiers. The output signals are fed into an acquisition system that digitizes and processes the data to extract energies and three-dimensional positions of the gamma-ray interactions.
The Compton camera's omnidirectional capability significantly increases gamma-ray sensitivity. A gamma ray enters the detector and interacts through Compton scattering until it is fully stopped by the photoelectric effect. Mathematical algorithms combining all of the gamma rays' energies $(E)$ at each position (p) are used to identify the path of the gamma-ray source. 
cannot detect the presence of oxygen because oxygen is stable and does not usually emit gamma rays. Stockpile stewardship and radioactive waste characterization may benefit from this capability. For example, the neutron spectrometer could measure oxygen bound to plutonium, which may have resulted from seepage through casks containing nuclear weapons or spent nuclear fuel. (See figure on p. 10.)

Physicist Stephan Friedrich of the Advanced Detector Group leads a team developing the ultrahigh-resolution neutron spectrometer. In addition to providing a spectral emission that distinguishes a light element within a heavy element matrix, neutron spectrometers can also detect nuclear material behind a shield and perhaps even determine the composition of the intervening material. If plutonium, for example, were concealed in a lead object, the lead, or any heavy metal shield, would absorb the gamma rays emitted from the plutonium, whereas neutrons would travel through the lead and scatter in ways that provide a signature.

The ultrahigh-resolution neutron spectrometer uses lithium fluoride to absorb incoming neutrons. It then measures the released energy from the neutrons interacting with the lithium fluoride combined with the energy of the neutrons as they enter the detector. The spectrometer will operate in laboratories where the units can be cooled to 0.1 kelvin,
The ultrahigh-resolution neutron spectrometer consists of a large cylindrical demagnetization refrigerator with a cold finger on one side, which contains a detector aimed at a radioactive source, and a box with preamplifiers on the other side. The lithium fluoride detector is held at a temperature of approximately 0.1 kelvin either in the refrigerator or in the cold finger. The rack on the bottom contains temperature control and dataacquisition electronics. Neutron spectra are displayed on the laptop computer.

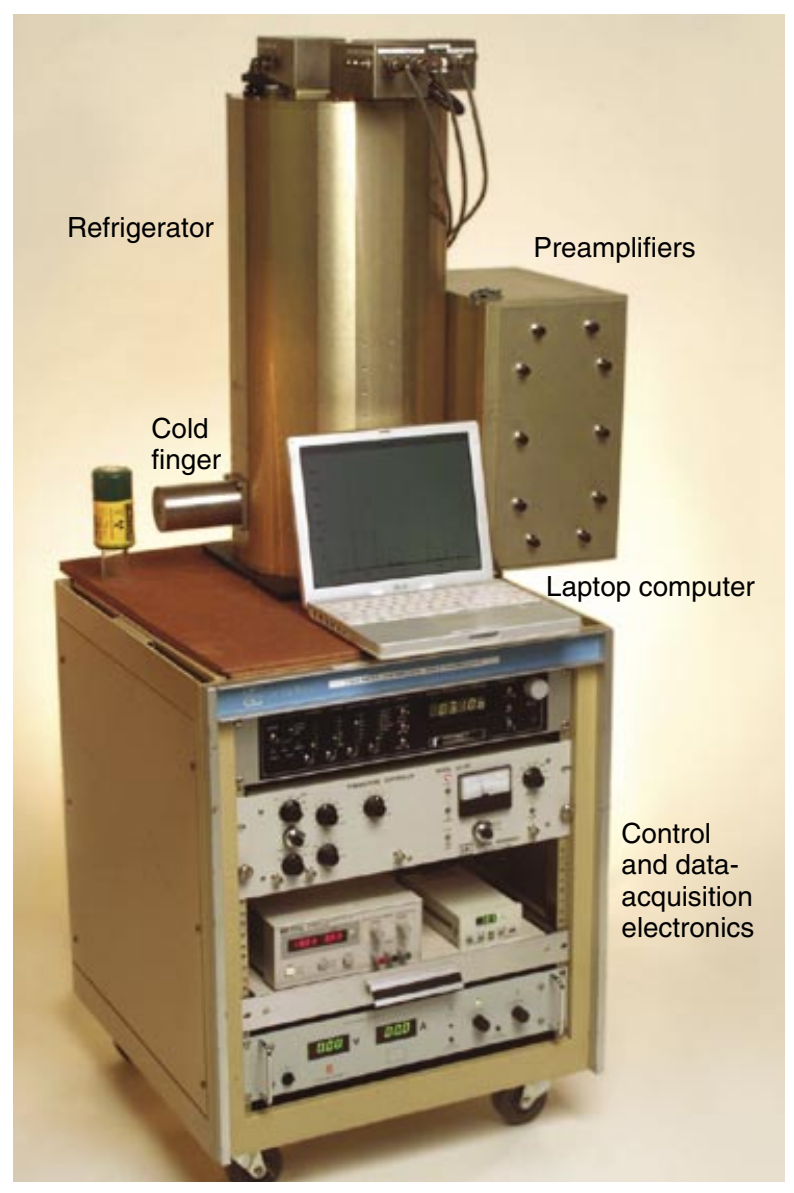

the temperature required to obtain ultrahigh-energy resolution.

"A prototype of the ultrahigh-resolution neutron spectrometer could be complete in a year," says Friedrich. "Collaborations at workshops conducted by the RDC helped our team refine ideas for this necessary technology and also revealed potential applications for other areas." The spectrometer is so sensitive that it will be able to detect the energy deposited by a single neutron.

\section{A New Frontier: Sensor Fusion}

Combining various types of radiation detection devices into a network that maximizes the benefits of each is the next frontier. Craig notes, "The first push was for radiation detectors. Now, there is the realization that a first responder called to check out a potential bomb threat on a bridge, for example, may not be in the best position to analyze the data." This realization has launched the area of sensor fusion - taking information from an ensemble of detectors.

"Sensor fusion," says Craig, "is a discipline that allows scientists to combine data and provide a sum greater than the parts. For example, information from a portable radiation detection system can be combined with that from handheld detectors and video cameras, and all of the data can be integrated to give a more complete report than one type of detector could provide."

Labov says DHS is interested in adding wireless data transmission to radiation detectors, but no system is currently in place to collect and analyze the data. "We could use analyses from science applications at the Laboratory to integrate and interpret the data. With sensor fusion, information gathered from multiple gamma-ray detectors, neutron detectors, and detectors that take visual images, for example, could be combined and analyzed together. Combining the information is a discipline in computer 
science. We are strategizing on the most effective methods to accomplish this goal."

\section{Sharing Bright Ideas}

Running analyses and interpreting the data from a network of different radiation detection equipment requires special skills in nuclear science. Yet despite the increasing demand for this expertise, the last two decades have seen a nationwide decline in the number of students pursuing careers in nuclear engineering, physics, and chemistry because of decreasing job prospects. For example, it has been decades since a nuclear power plant was built in the U.S. As a result, many universities are closing their nuclear energy programs and shutting down their research reactors.

But changes are under way. DOE is investing millions of dollars in the Nuclear Energy Research Initiative Program to support nuclear R\&D. The National Nuclear Security Administration (NNSA), the Department of Defense, and DHS also sponsor many R\&D efforts and are turning to Livermore to provide technical expertise in radiation detection. Jeff Richardson, a division leader for NAI, notes, "These agencies are looking to Livermore for creative solutions to homeland security and general nonproliferation objectives."

In 2000, the RDC formalized an outreach program to conduct workshops, coordinate technical discussion groups, and provide internships for university students interested in nuclear science. This year, the center hired an education coordinator to put together a plan for attracting new graduates to nuclear science. RDC members are working with students at universities, such as the University of California (UC) at Berkeley, which has a strong nuclear engineering program, and the University of Michigan, known for its nuclear science program. Graduate summer students work at Livermore's Seaborg Institute for Transactinium Science, which was established in 1991 to foster research in fundamental and applied nuclear science and technology.

Christie Shannon, the RDC's outreach coordinator, keeps a pulse on researchers' needs so that the center can arrange for relevant seminars and workshops. "The seminars and workshops provide a forum for collaboration with radiation experts within the Laboratory and from other organizations, including other national

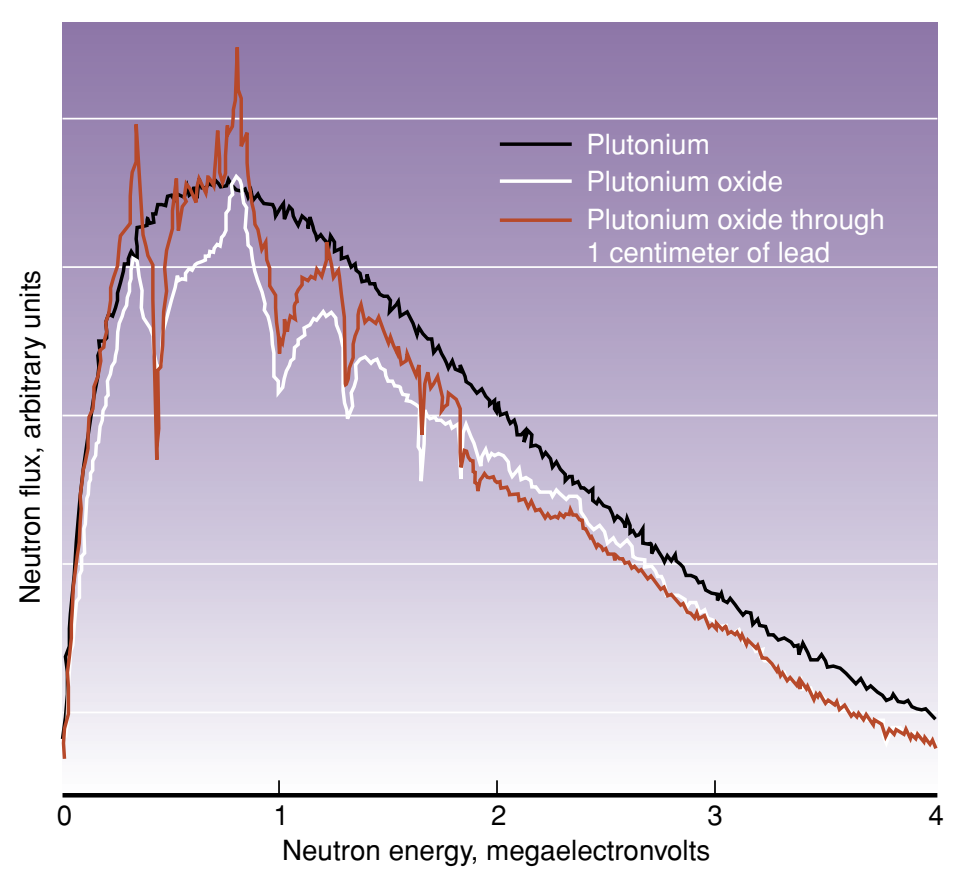

The ultrahighresolution neutron spectrometer can detect light elements, such as oxygen, within a matrix of heavy elements, such as plutonium. Shown here is a simulated neutron spectrum of plutonium, plutonium oxide, and plutonium oxide detected through lead.

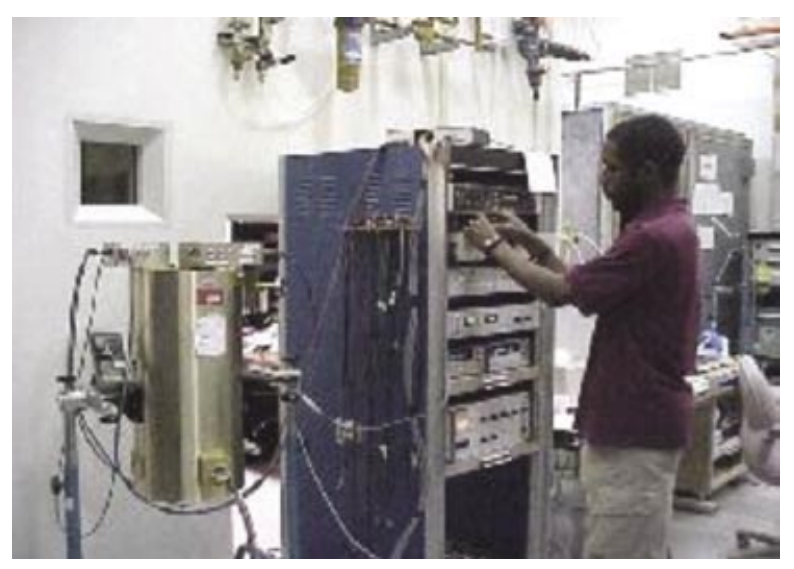

The Radiation Detection Center organizes internship programs for university students in nuclear science. Here, Kelvin Aaron from South Carolina State University operates a superconducting $x$-ray spectrometer. 
police cars to examine air particles after a nuclear terrorist attack.

In a series of workshops held from December 2003 to February 2004, 60 Laboratory scientists and engineers met to identify critical needs in radiation detection and prioritize a list of potential solutions. The idea for a large-scale effort in sensor fusion came from these collaborations. A new effort to develop detectors based on nanomaterials also emerged. A directional neutron detector and the ultrahigh-resolution neutron spectrometer are two other ideas that were refined at the workshops, enabling them to become fully funded R\&D projects. Friedrich notes, "After the workshops, the ultrahigh-resolution neutron spectrometer became a prominent goal for the Laboratory, and it became clear where we needed to go to complete development." The work on this spectrometer is now funded through NNSA's Office of Nonproliferation Research and Engineering.

The RDC also hosts DOE workshops featuring speakers with innovative ideas for detecting nuclear materials. Workshop topics have included investigating alternative signatures for detecting fissile materials; improving tags, seals, and intrusion sensors for container shipping security; and remotely monitoring nuclear material using attended and autonomous intelligent-sensor systems.

\section{Astrophysics: Past and Future}

Many Livermore radiation detection advances have resulted from technical developments in astrophysics. For years, Livermore researchers have collaborated with scientists from the California Institute of Technology, NASA's Goddard Space Flight Center, UC Berkeley, Columbia University, Massachusetts Institute of

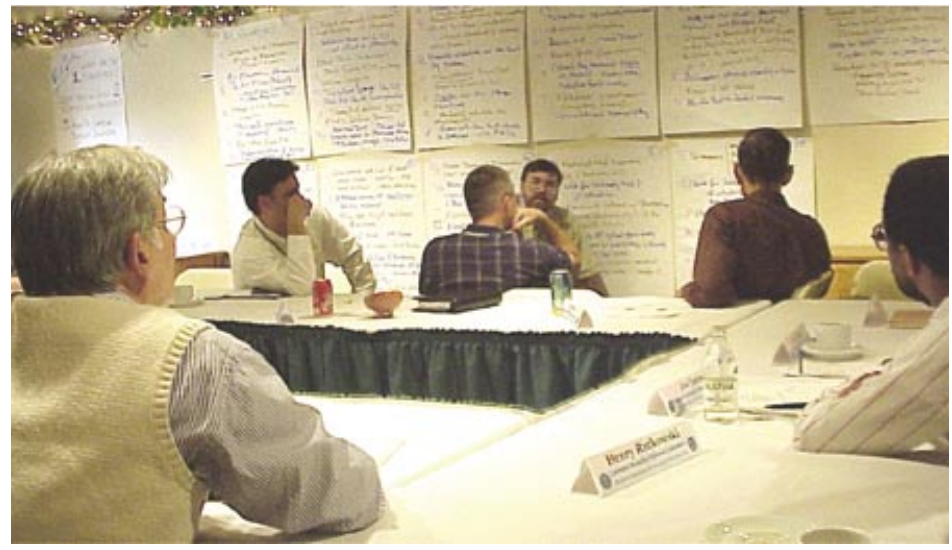

Workshops allow scientists and engineers to identify critical needs in radiation detection and share ideas for innovative solutions.

Technology, Harvard University, and other universities and government laboratories to develop the latest technologies for detecting and imaging space phenomena.

"We are finding ways to combine better science with better security," says Labov. "For example, astrophysicists at Harvard University approached Livermore for assistance on a satellite project in which the scientists needed an 8-square-meter area of crystal to operate as a detector. Some developments from that project resulted in technology later used for RadNet."

The coded aperture system used in GRIS is another example of a technology invented by astrophysicists. And the technology used for the Compton camera came from the Imaging Compton Telescope, COMPTEL, which was built in 1989 at Goddard to study gamma-ray sources such as pulsars, supernova remnants, and molecular clouds.

Although some of Livermore's radiation detection technology came from astrophysics, Craig hopes to see the reverse as well. "We're hoping to help astrophysicists use RadNet-like detection systems for missions, such as probing for black holes."
Detectors have come a long way over the last decade. From sensors on buoys to lowcost networks, today's devices promise almost tailor-made solutions to meet the needs of first responders. Richardson notes, "We are continuing to push the envelope in all areas of radiation detection, particularly in the areas of high sensitivity and selectivity."

As new tools for radiation detection and networking of systems develop, the RDC will continue to pilot efforts to realize the Laboratory's goals. Perhaps there may even be a new gadget for James Bond to take on his next mission.

\section{-Gabriele Rennie}

Key Words: Compton camera, gamma-ray imaging spectrometer (GRIS), large-area imager, Radiation Detection Center (RDC), RadNet, Seaborg Institute for Transactinium Science, sensor fusion, ultrahigh-resolution neutron spectrometer.

For further information contact Simon Labov (925) 423-3818 (labov1@IInl.gov) or visit rdc.IInl.gov.

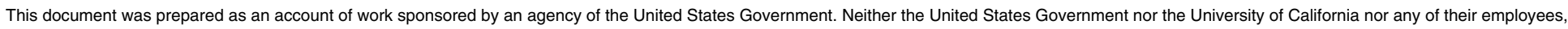

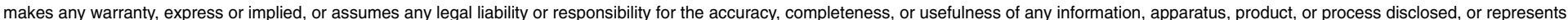

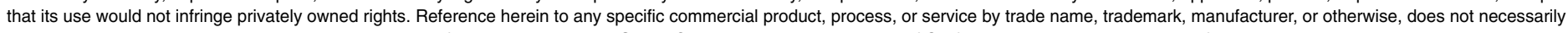

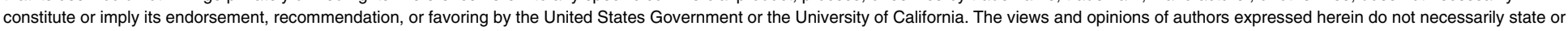
reflect those of the United States Government or the University of California, and shall not be used for advertising or product endorsement purposes.
}

This work was performed under the auspices of the U.S. Department of Energy by University of California, Lawrence Livermore National Laboratory under Contract W-7405-Eng-48. 